\author{
Vol.1 No.2, Summer 2020
}

\title{
The effect of combined decongestive therapy and pneumatic compression pump on body image in women with breast cancer related lymphedema
}

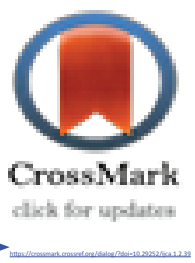

\author{
Jaafari $\mathrm{B}^{1^{*}}$, Hashemifard $\mathrm{T}^{2}$, Mehri $\mathrm{A}^{3}$
}

1- Department of Nursing and Midwifery, Kazerun Branch, Islamic Azad University, Kazerun, Iran .

2- Assistant Professor, Department Nursing and Midwifery, Kazerun Branch, Islamic Azad University, Kazerun, Iran.

3- Assistant Professor, Department of Health Education, School of Health, Sabzevar University of Medical Sciences, Sabzevar, Iran.

Corresponding author: Jaafari B, Department of Nursing and Midwifery, Kazerun Branch, Islamic Azad University, Kazerun, Iran .

Email: badrijaafari@kau.ac.ir

Received: 26 January 2019

Accepted: 3 July 2019

\begin{abstract}
Introduction: Patients with breast cancer who have two positive axillary lymph nodes, along with mastectomy, they undergo axillary node dissection. Lymphedema after axillary surgery is a feared complication. This women experience pain and body image impairments. Any intervention to reduce lymphedema, affects the body image of these patients.

Method: This study is a randomized, single-blind clinical trial that 112 women with breast cancer related lymphedema participated. After collecting data through a questionnaire of body image and relationships scale, the community was randomly divided in to experimental (54) and control $(n=58)$ groups. For the experimental group, Combined Decongestive Therapy and pneumatic compression pump was performed by an educated nurse, twice a week for six consecutive months. The control group was placed on the waiting list to be treated after the end of intervention in the experimental group. At the end of intervention, two groups completed the questionnaire again. Data was analyzed in SPSS software (version 17).

Results: After the intervention the mean score of health and strength, social barriers and appearance and sexuality as well as total score in the experimental group was significantly higher than the control group, which was significant except for social barriers in the other factors $(p<0.05)$. The highest mean increase was observed in the health and strength fact.

Conclusion: The results showed that combined decongestive therapy and compression pump are effective on the body image and social interactions in the patients, which affects the body image of patients more than their social interactions. Therefore, combined decongestive therapy and pneumatic compression pump along with psychological interventions are recommended to reduce the physical and psychological complications in these patients.

Key words: Lymphedeme, Breast Cancer, Body Image, Combined Decongestive Therapy.
\end{abstract}

\begin{tabular}{|l|l|}
\hline \multicolumn{3}{|c|}{ Access this article online } \\
\hline
\end{tabular}




\title{
تأثير احتقان زدايَ تركيبى و يمٍٍ فشارى بر تصوير ذهنى در زنان مبتلا به تورم لنفاوى ثانويه به درمان سرطان يستان
}

\author{
بدرى جعفرى "'، طاهره هاشمى فرد"، على مهرى"
}

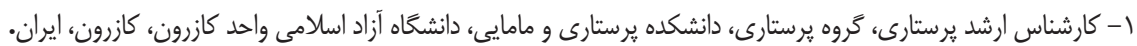

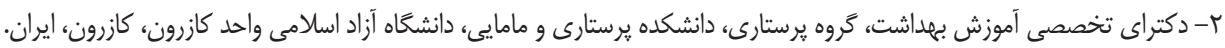

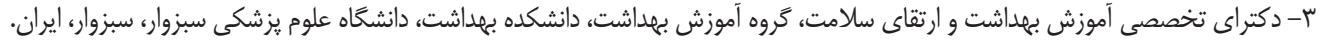

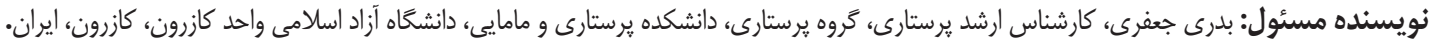

ايميل: badrijaafari@kau.ac.ir

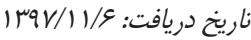

مقدمه: بيماران مبتلا به سرطان يستان در صورتى كه دو غده لنفاوى زير بغل آنها مثبت باشد، همزمان با ماستكتومى تحت جراحى برداشتن غدد لنفاوى زير بغل قرار مى گيرند. تورم لنفاوى بعد از جراحى زير بغل، يك عارضه ترسناك است. مبتلايان به تورم لنفاوى درد و مشكلات

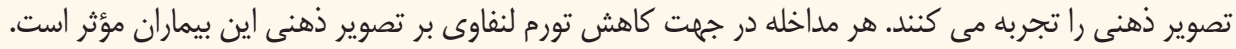

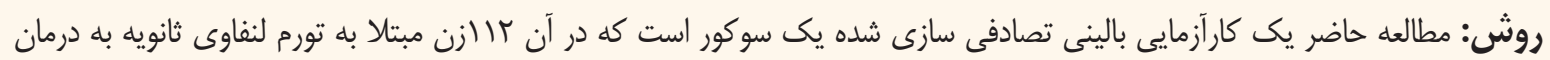

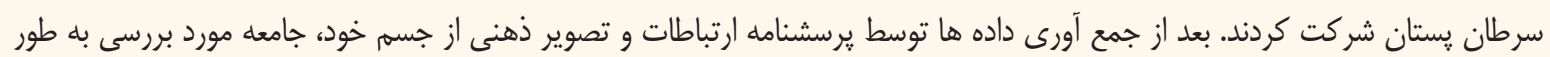

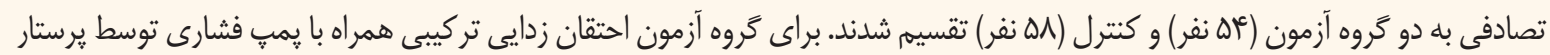

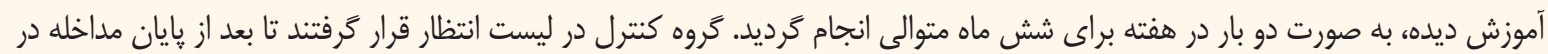

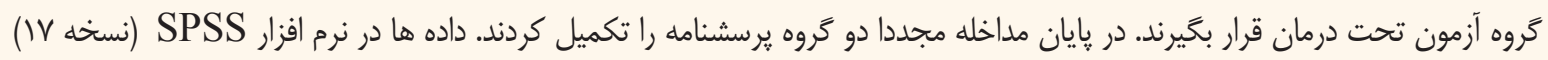
مورد تجزيه و تحليل قرار گرفت.

يافته ها: ميانكَين نمره سلامت و قدرت، موانع اجتماعى، ظاهر و جذابيت جنسيتى و همجنين نمره كل بعد از مدان مداخله در كروه آزمون نسبت

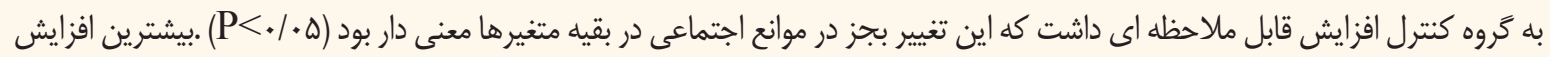
ميانكين در متغير سلامت و قدرت مشاهده گرديد. نتيجه كَيرى: احتقان زدايى تركيبى و يمب فشارى بر تصوير ذهنى و تعاملات اجتماعى مبتلايان به تورم لنفاوى مؤثر است و اين تاثير بر

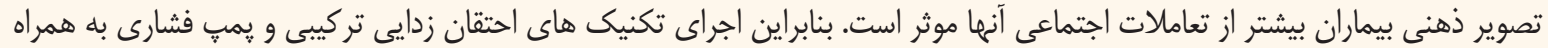

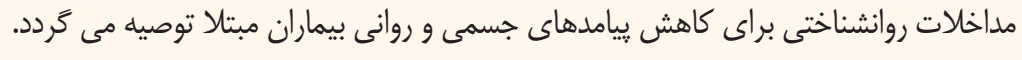

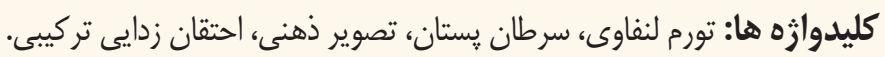

مشكلات بهداشتى عبارتند از بيماريهاى مزمن مانند تورم لنفاوى كه مقلدمه ناشى از درمان سرطان يستان مى باشند و عملكرد و كيفيت زندگى اين بيماران را تحت تأثير قرار مى دهند (V). تقريبا از هر ينج زن

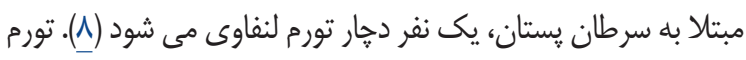

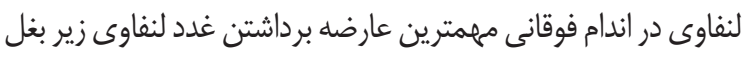
و درمان هاى سرطان يستان است كه در نتيجه اختلال در مسيرهاى ئى تخليه مايع لنف ايجاد مى گردد. تجمع مايع لنف سرشار از يروتئين در بافت هاى زير جلدى فرد را مستعد ابتلا به عفونت مى كند. درمان سرطان پِّتان شايعترين كانسر در زنان است (ب-1). در ايران نيز

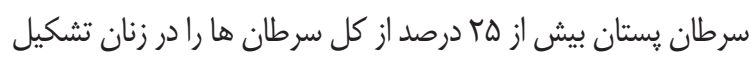

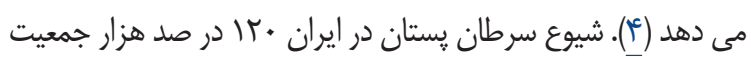

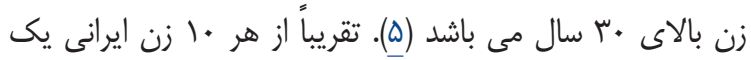

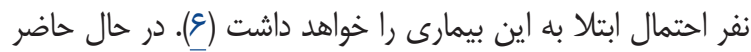
درمانگران به مشكلات بهداشتى بيماران مبتلا به سرطان پستان، جهت افزايش ميزان بقاء آنها بيشتر از كذشته توجه مى كنند. اين 
نمونه ها براى هر گروه • لنفر تعيين گَرديد. سبس بيماران واجد شرايط بطور تصادفى بصورت يك در ميان به دو كروه كنترل و

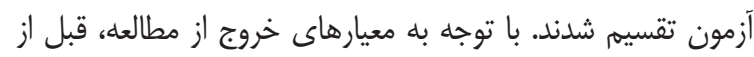

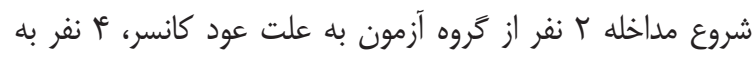

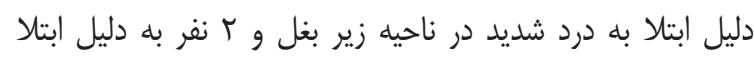

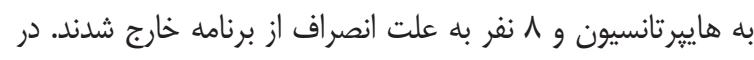

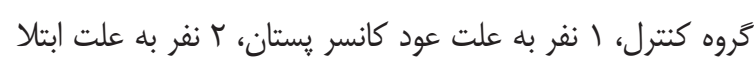

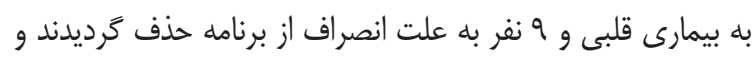

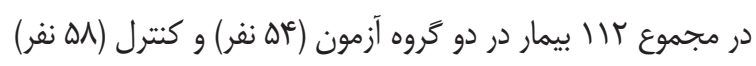
شر كت داشتند. از تمام بيماران درخواست كَرديد كه در صورت تمايل به شركت در مطالعه رضايت نامه كتبى را امضاء كنند.

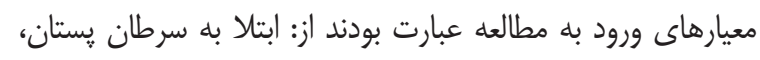
سابقه جراحى و شيمى درمانى و در صورت نياز هورمون درمانى

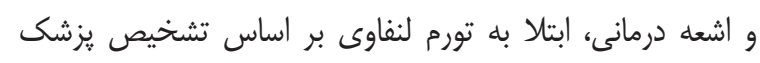

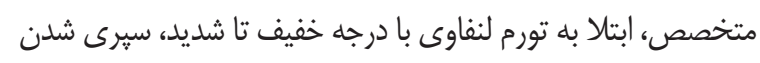

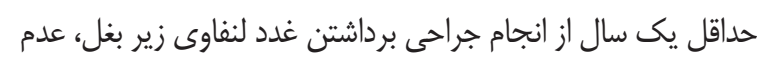

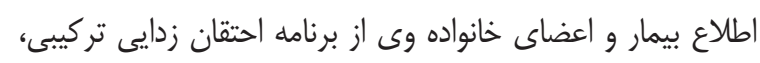
دسترسى به تلفن، داراى سن بين •1 ـ ع آس سال بود.

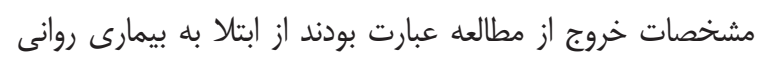

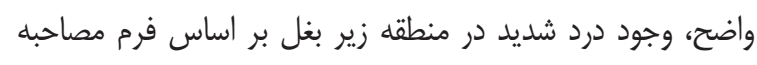

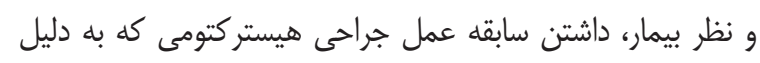

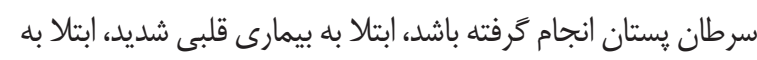

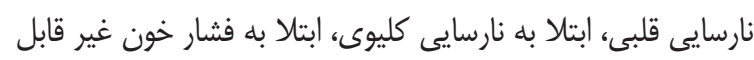

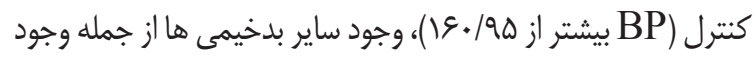

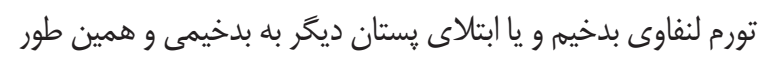

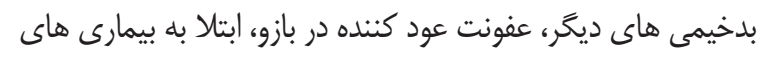

ماهيجه اى و مفصلى. قبل از شروع مداخله دو كروه از نظر تعداد غدد لنفاوى مثبت ماند (P/DVY)

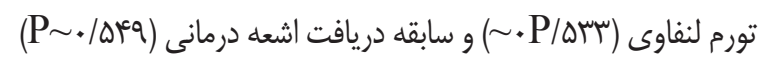

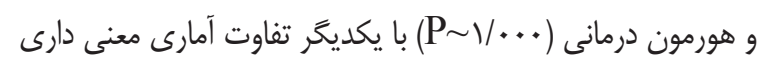
نداشتند (جدول). قبل از شروع مداخله همه بيماران يرسشنامه (برانه ارتباطات و تصوير ذهنى از جسم خود را تكميل كردند.
تورم لنفاوى دشوار است و نياز به فيزيك درمانى طولانى مدت يا

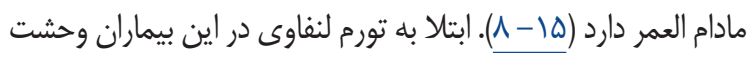

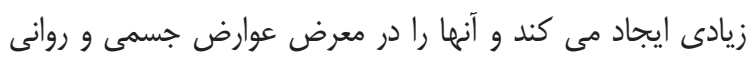

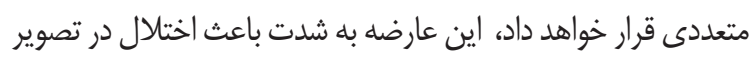

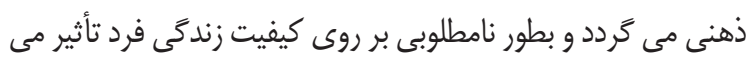

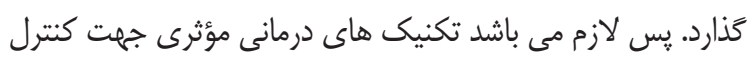
تورم لنفاوى مرتبط با درمان هاى سرطان يستان و كاهش إندان اثرات

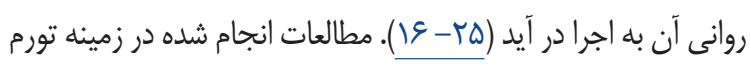

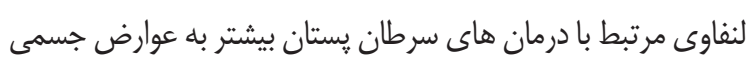

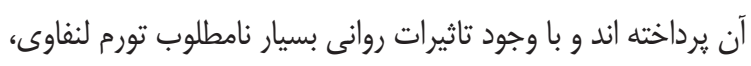

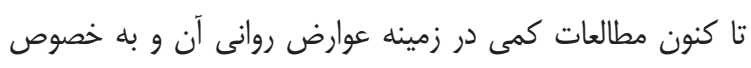
اختلال در تصوير ذهنى و محدوديت هاى تعاملى اين بيمار ان صورت كرفته است (ب) (II). احتقان زدايى تركيبى و بمب فشارى به عنوان يكى از مداخلات

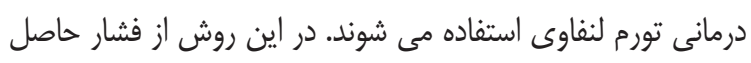

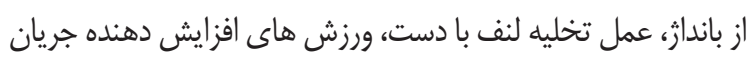

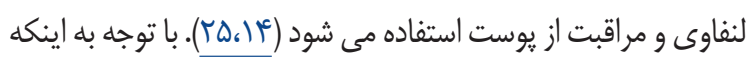
كنترل و كاهش تورم لنفاوى مى تواند منجر به بهبود تصوير ذهنى و

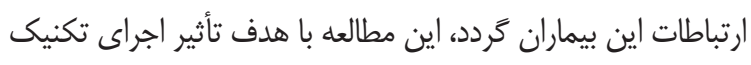

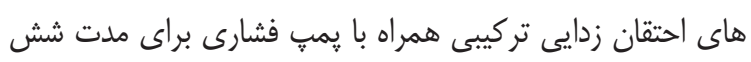

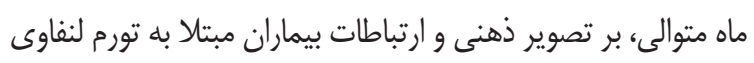

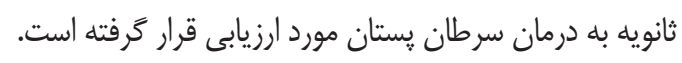

\section{روش مطالعه}

مطالعه حاضر يك كار آزمايى بالينى تصادفى سازى شده يك سوكور

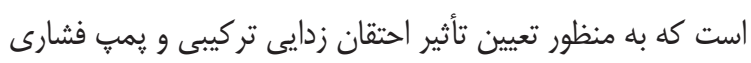
بر تصوير ذهنى در بيماران مبتلا به تورم لنفاوى ثانويه به درمان

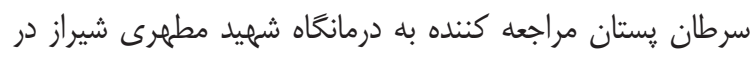

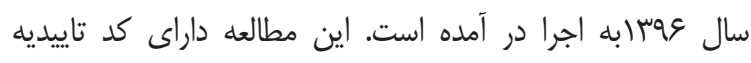
IRCT138902212621N8

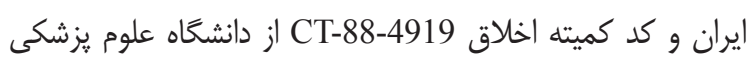

$$
\text { شيراز مى باشد. }
$$

جهت تعيين حجم نمونه كافى از روش مطالعه مشابه (عَ) استفاده

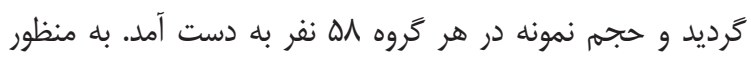

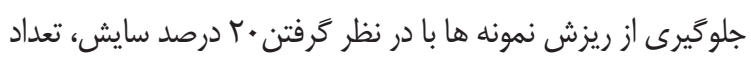


جدول (: مقايسه دو گروه در شروع مداخله

\begin{tabular}{|c|c|c|c|}
\hline p-value & كروه كنترل (N= & كَروه آزمون (N= OF) & \\
\hline.$/ \Delta$ & $r / q \pm V / \Delta q$ & $\Gamma / \Gamma \Lambda \pm \Gamma / \varsigma$ & تعداد غدد لنفاوى برداشته شده \\
\hline .10 & $\begin{array}{l}1(1 F) \\
1 F(T F) \\
11(19)\end{array}$ & 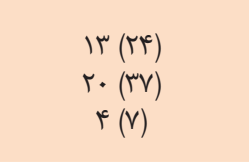 & 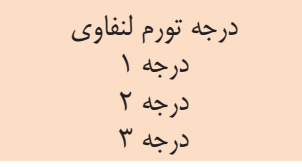 \\
\hline.$/ \Delta$ & 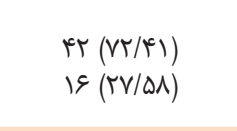 & $\begin{array}{l}r \cdot(V c / \cdot V) \\
\text { If }(r \Delta / q r)\end{array}$ & دست دست درَير \\
\hline $\begin{array}{c}1 \\
.19 \\
1\end{array}$ & $\begin{array}{l}\operatorname{C\Delta }(V V / \Delta \Lambda) \\
\Delta r(\Lambda ৭ / \& \Delta) \\
\Delta \Lambda(1 \cdots)\end{array}$ & $\begin{array}{l}\operatorname{Rr}(\Lambda 1 / \mathbb{R} \wedge) \\
\operatorname{ar}(q \varepsilon / \mathrm{rq}) \\
\operatorname{\Delta r}(1 \cdots)\end{array}$ & شورمون درمانى \\
\hline
\end{tabular}

مجدداً اين كار انجام گرديد. به اين ترتيب ضريب همبستخى دو بار

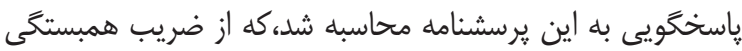

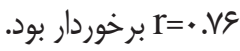

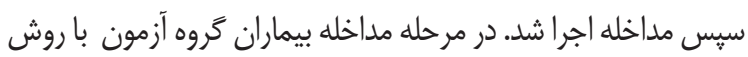
احتقان زدايى تركيبى همراه با يمٍ فشارى تحت درمان قرار گرفتند

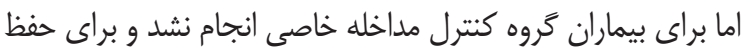
ضوابط اخلاقى از آنها ثبت نام بعمل آمد و در نوبت انتظار قرار گرفتند

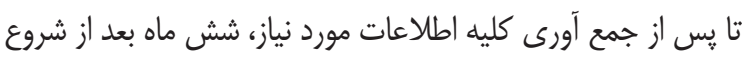

مداخله، تحت درمان با احتقان زدايى تركيبى قرار گَيرند.

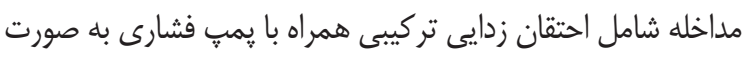

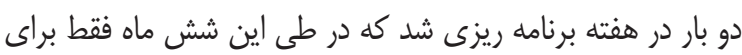

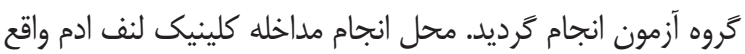
در درمانگاه شهيد مطررى شيراز بود. هر جلسه احتقان زدايى تركيبى

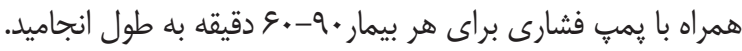

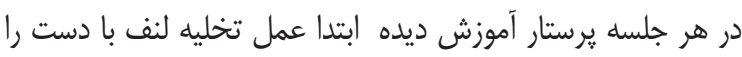

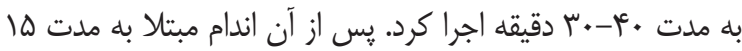
دقيقه در داخل پمب فشارى قرار گرفت. سيس دست بيمار با استفاده

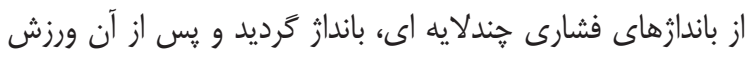
هاى درمانى به منظور افزايش جريان مايع لنف براى بيمار انجام شد. طى اين جلسات براى گروه آزمون، آموزش هاى كتبى و شفاهى در

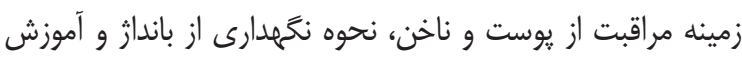

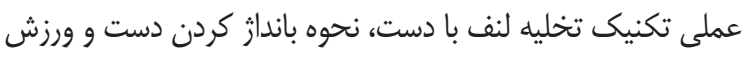

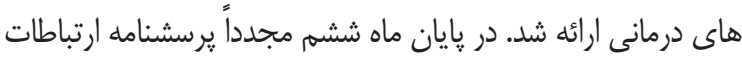
و تصوير ذهنى از جسم خود در اختيار بيماران در دو گروه آزمون و كنترل قرار گرفت تا آن را تكميل كنند. سيس براى بررسى تاثير مداخله بر تصوير ذهنى بيماران، تغييرات
يرسشنامه ارتباطات و تصوير ذهنى از جسم خود، يك يرسشنامه متشكل از rس سوال است كه از سه بخش يا عامل تشكيل شده

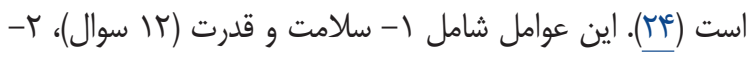

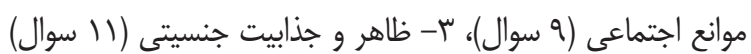
هستند. عامل اول اختلال فيزيكى درى شده در ارتباط با دران درمان

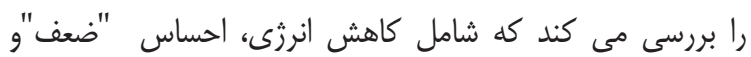

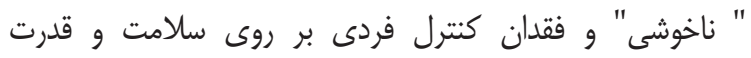

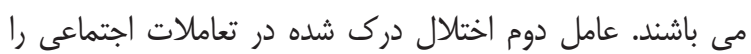
بررسى مى كند كه شامل كاهش فعاليت هاى اجتماعى ناشى از درمان سرطان گِتان و نخَرانى هاى مربوط به علائم روانى و

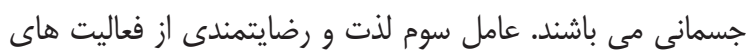

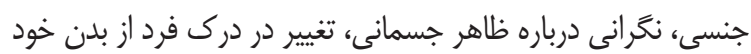

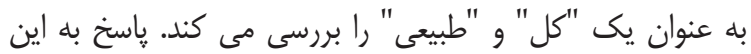
جملات بر مبناى يك مقياس درجه بندى ه- نقطه اى دو سويه نمره

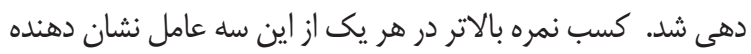
اختلال بيشتر در آن عامل بود.

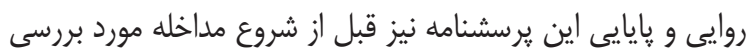

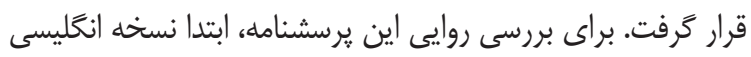

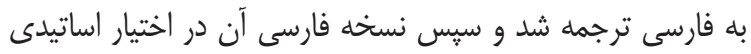
قرار گرفت كه در اين زمينه تخصص داشتند. سپيس طبق نظرات

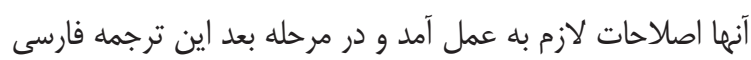

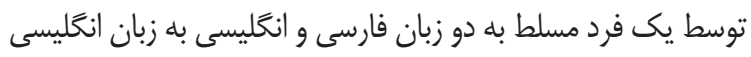
بازگر دانده شد كه تفاوت جندانى با متن انخَليسى ابتدايى نداشت. براى بررسى پايايى اين پرسشنامه، از روش بازآزمايى استفاده شد.

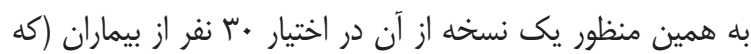
معيار ورود به مطالعه را داشتند) قرار گرفت و سيس دو هفته بعد 


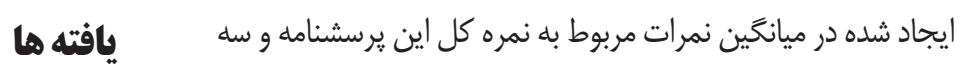

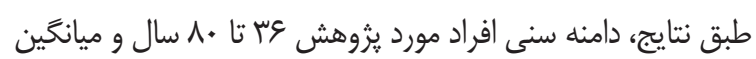

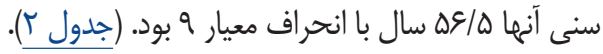

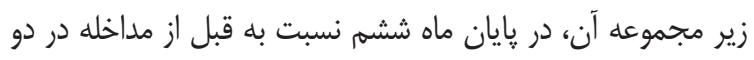
كروه مورد مقايسه قرار كرفت. تحليل داده ها با استفاده از نرم افزار SPSS، نسخه لا و و با استفاده

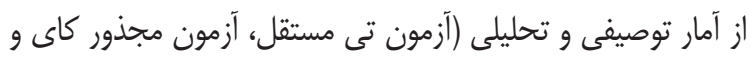

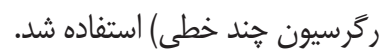
جدول ז: مشخصات بيماران در كروه آزمون و كروه كتترل

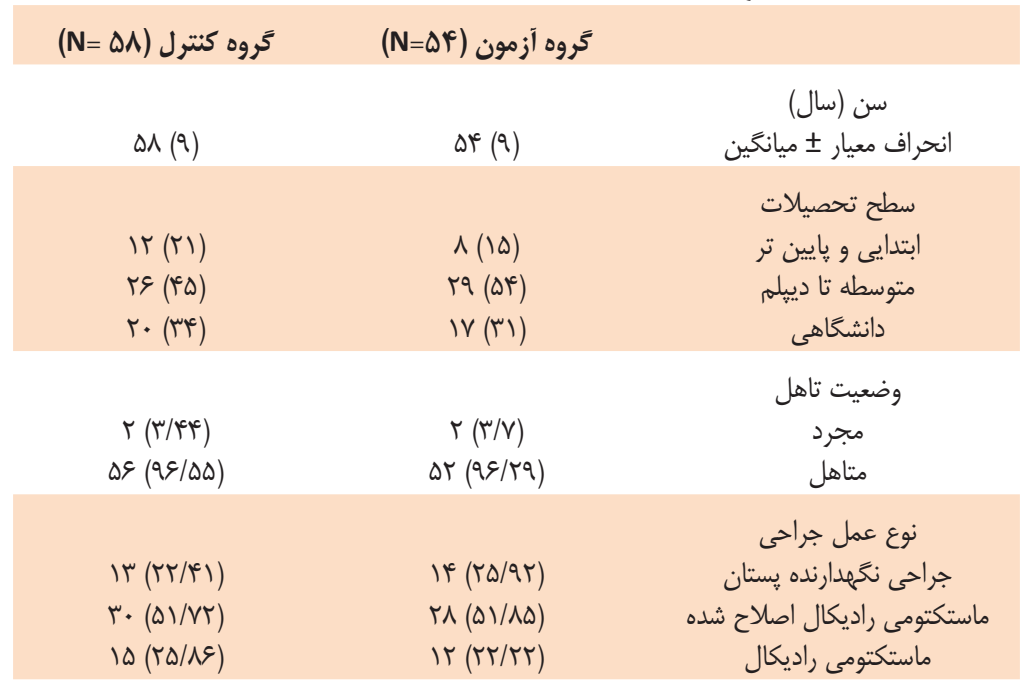

جنسيتى و همجنين نمره كل بعد از مداخله در كروه آزمون نسبت ميانگين نمرات حاصل از يرسشنامه تصوير ذهنى از جسم خود و

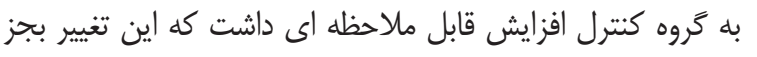

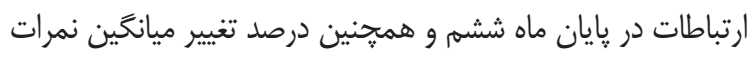

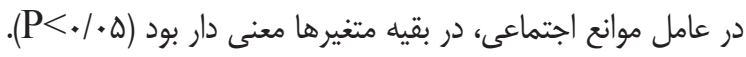

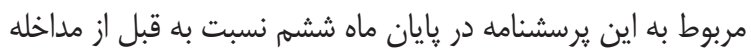
بيشترين افز ايش ميانكَين در عامل سلامت و قدرت مشاهده كَرديد. مربوط به كَروه آزمون و كروه كنترل در (جدولَّ) نشان داده شده است. ميانكين نمره سلامت وقدرت، موانع اجتماعى وظاهر و جذابيت جدول ب: مقايسه دو كروه آزمون و كنترل از نظر ميانكين نمرات حاصل از يرسشنامه تصوير ذهنى از جسم خود و ارتباطات در يايان ماه ششمه و همجنين درصد

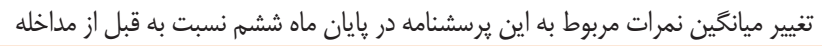

\begin{tabular}{|c|c|c|c|c|c|}
\hline \multirow[b]{2}{*}{ سطن دماري } & \multicolumn{2}{|c|}{ تروه كنترل (N = } & \multicolumn{2}{|c|}{ كروه آزمون (N = DF) } & \multirow[b]{2}{*}{ متغيرها } \\
\hline & 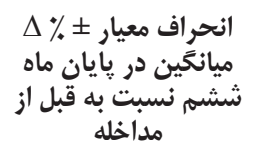 & 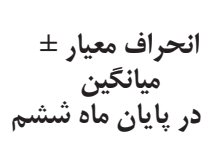 & 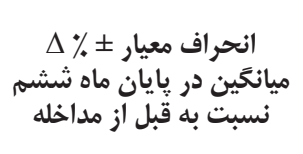 & 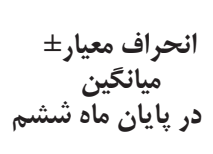 & \\
\hline$<\cdot / \cdots)$ & $-\cdot / 4 \pm \mid f / \pi$ & $V N / \cdot \pm I N / r$ & $\mid r / \cdot \pm I N / T$ & $V \cdot 1 \cdot \pm 19 / \Delta$ & نمره كل \\
\hline$<\cdot 1 \cdots+$ & $-\cdot / r \pm 18 / 9$ & $\mathrm{r} / \Lambda \pm N / \Lambda$ & $1 Q / V \pm T E / V$ & $r V / V \pm q / r$ & سلامت و قدرت \\
\hline.$/ \mathrm{V}$ & $-r / \Delta \pm r V / l$ & $\mid \omega / \Lambda \pm \xi / \Delta$ & $s / \Delta \pm M F / \Lambda$ & $\| f / V \pm \Delta / \Lambda$ & تعاملات اجتماعى \\
\hline $.1 \cdot 4$ & $-1 / f \pm 19 / V$ & $r N / \Lambda \pm g / r$ & $V / 9 \pm 1 N / 9$ & $\mathrm{rV} / \mathrm{\Lambda} \pm \mathrm{V} / \cdot$ & ظاهر و جذابيت جنسيتى \\
\hline
\end{tabular}

معنى دار در نمره حاصل از عامل تعامل اجتماعى تردد. اين عامل

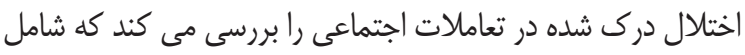

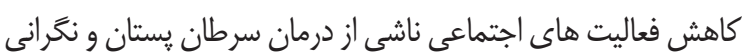
هاى مربوط به علائم روانى و جسمانى مى باشند. دليل دستيابى به اله

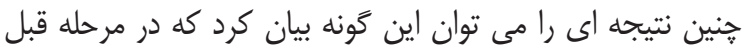

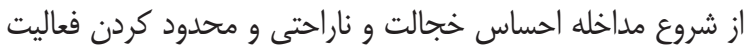
در حال حاضر احتقان زدايى تركيبى يك روش درمان محافظتى

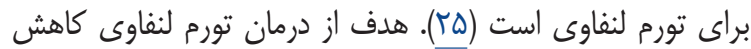

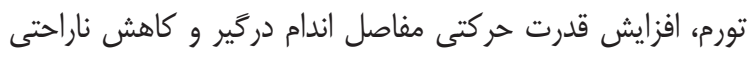

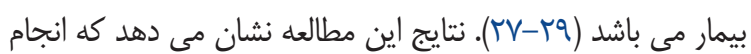
مداخله طى مدت شش ماه نتوانست منجر به ايجاد تغييرات آمارى 
فرد از ظاهر و جذابيت جنسيتى خود بهبود مى يابد، همانطور كه در يزوهش حاضر اجراى تكنيك هاى احتقان زدايى تركيبى باعث ايجاد

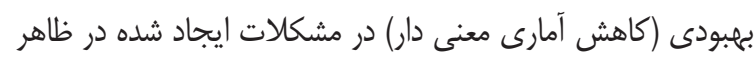

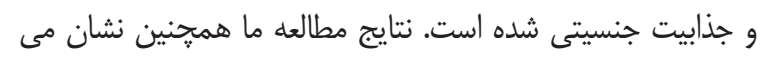

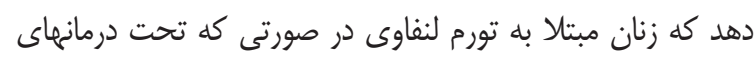

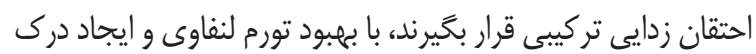

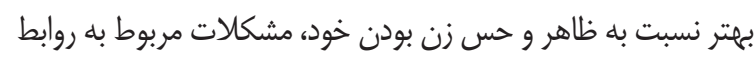

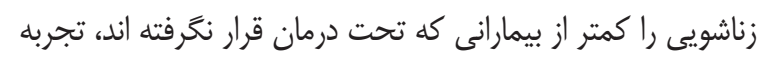

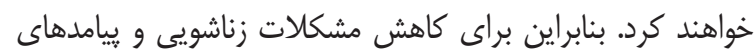
آن اجراى تكنيك هاى احتقان زدايى تركيبى در زنان مبتلا به تورم لنفاوى توصيه مى شود.

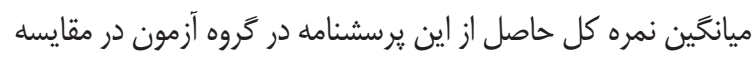
با كروه كنترل كاهش آمارى معنى دارى ييدا كرده است. بـ به دئ دليل

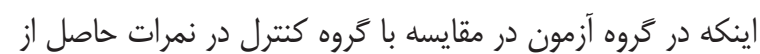

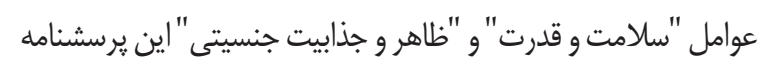

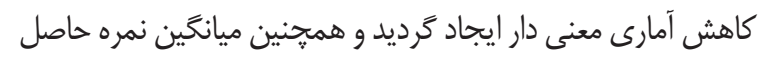

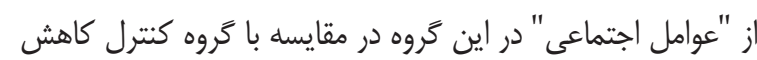

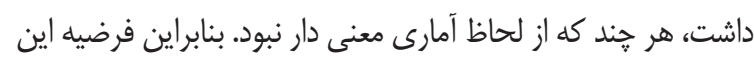

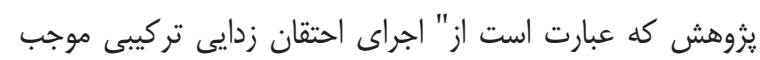

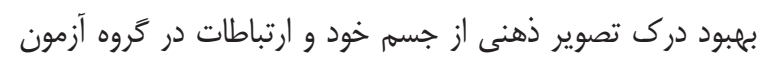

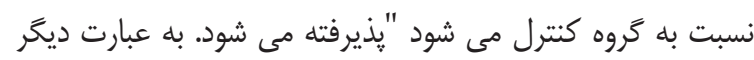

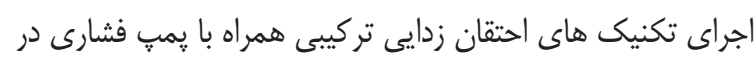

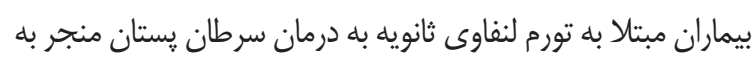
كاهش تورم لنفاوى و در نتيجه بهبود تصوير ذهنى از جسم خود و

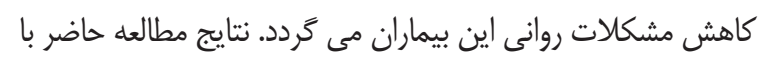

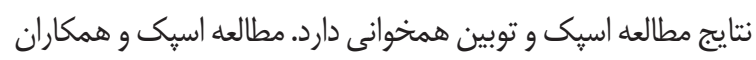

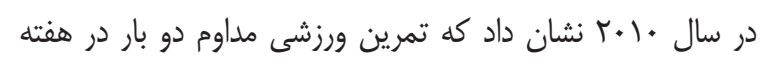

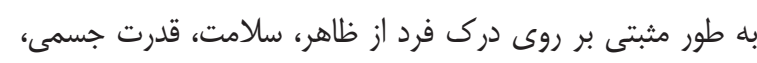

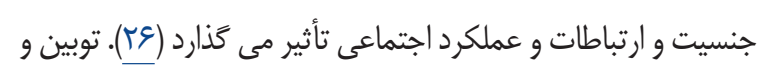

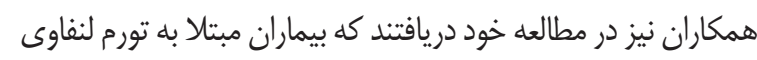

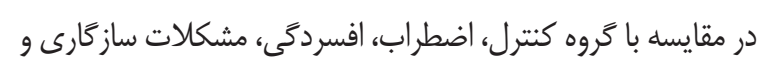
مشكلات شغلى، خانوادگى، اجتماعى و جنسى بيشترى دارند. بعلاوه

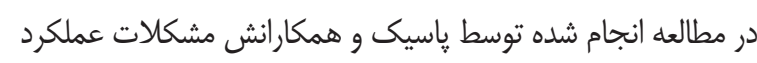

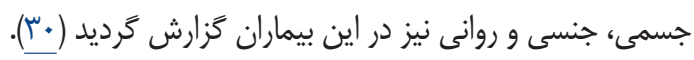

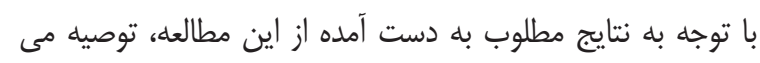

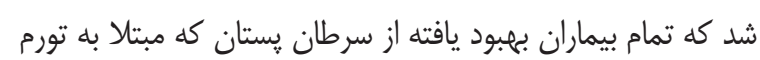

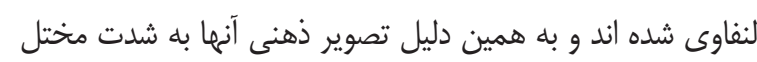

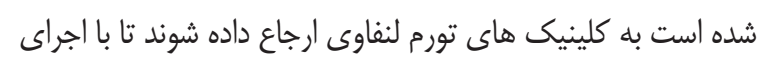

هاى اجتماعى و نيز خوددارى از انجام فعاليتهاى مورد علاقه به دليل

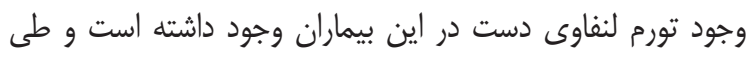

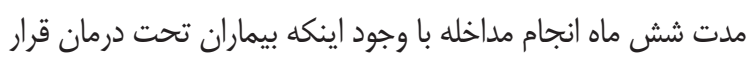

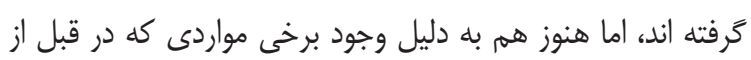

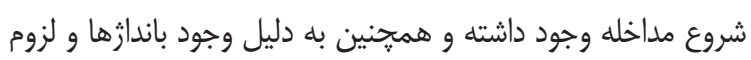

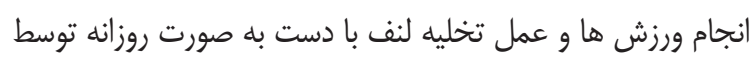
خود بيماران در منزل باعث شده است كه تعاملات اجتماعى فرد در

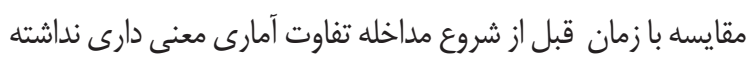

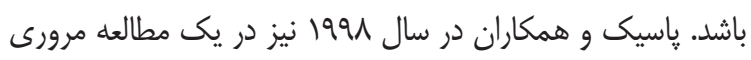
كزارش كردند كه در اكثر زنان وجود باندازهاى فشارى مى توان تواند

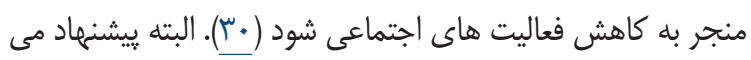

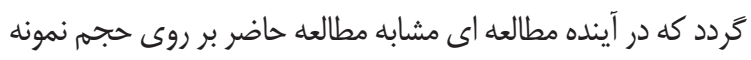

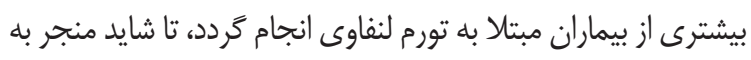

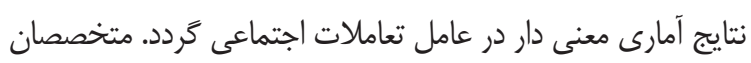

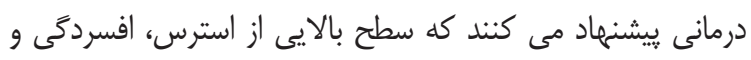

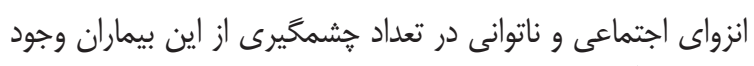

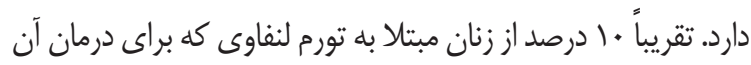

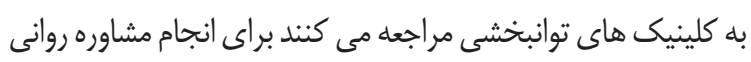

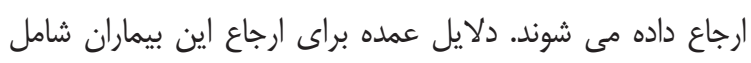

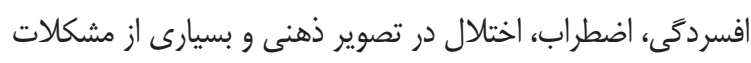

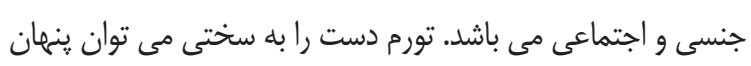

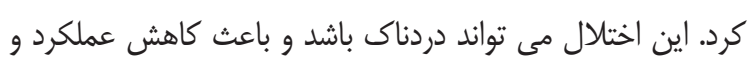

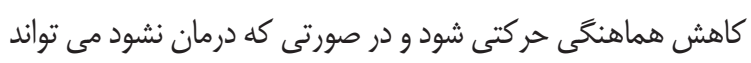

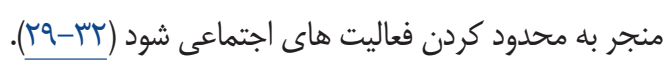

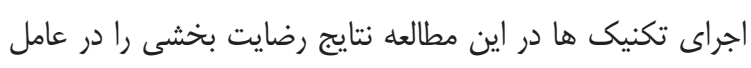

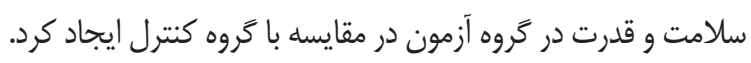

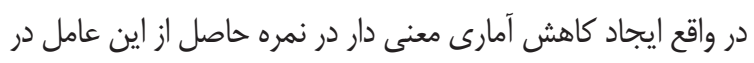

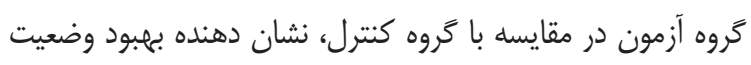
جسمانى و كاهش احساس خستخى بيماران اين كروه و در نهايت

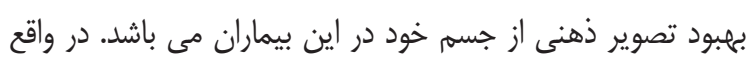

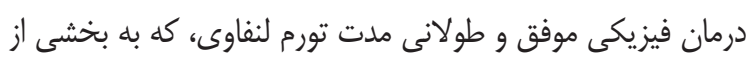

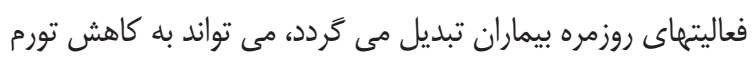

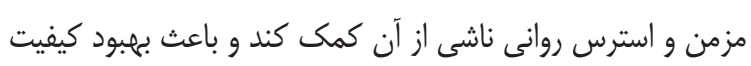
زندخى بيمار شود (Iآ). ميانكَين تغييرات نمره حاصل از عامل (ظاهر و جذابيت جنسيتى)

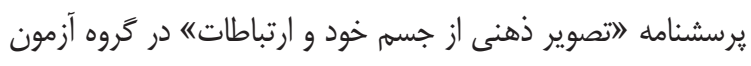

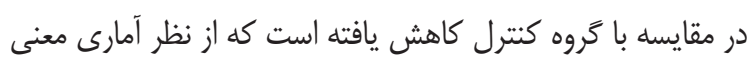

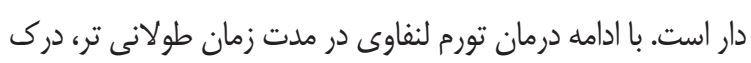




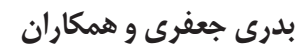

بدينوسيله محققان مراتب تشكر و قدردانى صميمانه خود را از مديريت و يرسنل محترم كلينيك لنف ادم درمانكاه مطهرى شيراز كه در طول مدت انجام يثوهش حاضر نهايت همكارى را با آنان

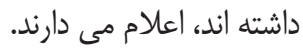

\section{References}

1. Soderman M, Friberg E, Alexanderson K, Wennman-Larsen A. Women's experiences of encounters with healthcare professionals' regarding work after breast-cancer surgery and associations with sickness absence: a 2 - year follow-up cohort study. 2019, April; 27 (10): 1197-206.

2. Vignes S, Prudhomot PF, Simon L, SanchezBrechot ML, Arrault M, Locher F. Impact of breast cancer- related lymphedema on working women. Supportive Care in Cancer, 2019, April; 1-7.

3. Fauci A, Braunwald E, Kasper Hauser S, Logo D, Jameson J, Loscalzo J. HARRISON'S: manual of medicine. 17th ed. Philaddelphia; 2009.

4. Taleghani F, Yekta ZP, Nasrabadi AN. Coping with breast cancer in newly diagnosed Iranian women. J Adv Nurs, 2006; 54 (3): 265-73.

5. Cancer office of disease management center and cancer research center of cancer institute. Abstracts of second nation wide breast cancer congress. Tehran: 2006: 30 [in persian].

6. Montazeri A, Ebrahimi M, Mehrdad N, Ansari M, Sajadian A. Delayed presentation in breast cancer: A study in Iranian women. Bio MED central women Health, 2003; 3 (1): 40.

7. Ahmed RL. Physical activity and lymphedema (the PAL trial): Assessing the safety of progressive strength training in breast cancer survivors. Contemp Clin Trials, 2009, May; 30 (3): $233-45$.

8. Rupp J, Hadamitzky C, Henkenberens C, Christiansen H, Steinmann D, Bruns F. Frequency

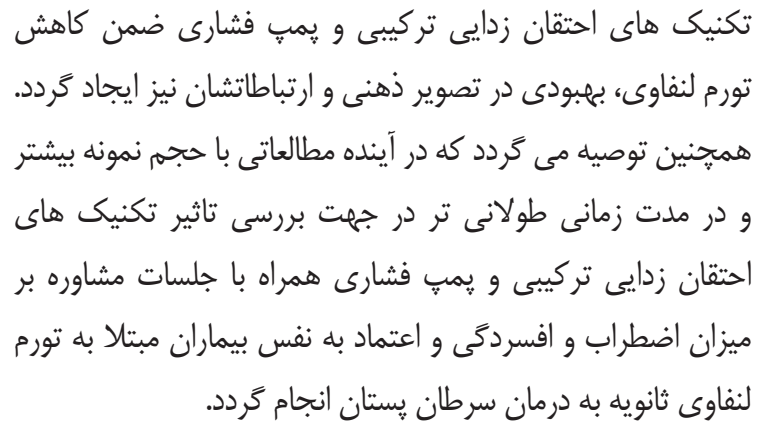

\section{نتيجه كيرى}

نتايج نشان داد كه احتقان زدايى تركيبى و پِي فشارى بر تصوير

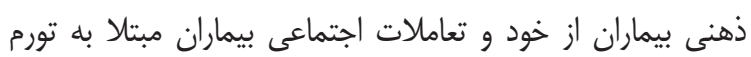

and risk factors for arm lymphedema after multimodal breast-conserving treatment of nodal positive breast Cancer - a long-term observation. Radiat Oncol, 2019, Mar 7; 14 (1): 39.

9. Isaksson G, Feuk B. Morbidity from axillary treatment in breast cancer- a follow-up study in a district hospital. Acta Oncol, 2000; 39: 335-6.

10. Borman P, Yaman A, Denizli M, Karahan $\mathrm{S}$, Ozdemir O. The reliability and validity of lymphedema quality of life questionare- Arm in the Turkish patients with upper limb lymphedema related with breast cancer. Turk J Phys Med Rehab, 2018; 64 (3): 205-12.

11. Sen Y, Koelmeyer L, Brotkanics R, Ricketts R, Mackie H, C.Lan T, et al. Breast Cancer Related Lymphedema: Differentiating Fat from Fluid Using Magnetic Resonance Imaging Segmentation. LYMPHTIC RESEARCH AND BIOLOGY, 2017; 00 (00): 1-8.

12. Chang DW, Masia J, Gorza R, Skoracki R, Neligan PC. Lymphedema: Surgical and Medical Therapy. Plastic and Reconstructive Surgery, 2016, September; 138 (35): 2095 -185.

13. Lewis-Smith H, Claire Diedrichs P, Harcourt D. A pilot study of a body image intervention for breast cancer survivors. Body Image, 2018, December; 27: 21-31.

14. Kaviani A, Lotfi M. Control of lymphedema after breast cancer treatment. 1st ed. Tehran: Tehran university of medical sciences; 2006: 13-74 [in Persian].

15. Golshan M, Smith B. Prevention and Management of Arm lymphedem in the Patient with Breast Cancer. J Support Oncol, 2006, Sep; 
4 (8): 381-6.

16. Temur K, Kapucu S. The effectiveness of lymphedema self-management in the prevention of breast cancer-related lymphedema and quality of life: A randomized controlled trial. European Journal of Oncology Nursing, 2019, Jun; 40: 2235.

17. Alcorso J. Sherman KA. Factors associated with psychological distress in women with breast cancer-related lymphedema. Psycho-oncology, 2016, Jul; 25 (7): 865 - 72.

18. Nielsen I, Gordon S, Selby A. Breast cancerrelated lymphoedema risk advice: a challenge reduction for health professionals. Cancer Treat Rev, 2008; 34 (7): 621-8.

19. Baron RH, Kelvin JF, Bookbinder M, Cramer L,Borgen PI, Thaler HT. Patient s sensations after breast cancer surgery. Apilot study. Cancer Pract, 2000; 8 (5): 215-22.

20. Nesvold IL, Dahl AA, Lokkevik E, Marit Mengshoel A, Fossa SD. Arm and shoulder morbidity in breast cancer patients after breastconserving therapy versus mastectomy. Acta Oncol, 2008; 47 (5): 835-42.

21. Lopez E, Nunez MI, Guerrero MR, del Moral $\mathrm{R}$, de Dios Luna J, del Mar Rodriguez M, et al. Breast cancer acute radiotherapy morbidity evaluated by different scoring systems. Breast Cancer Treat, 2002; 73 (2): 127-34.

22. Ochlek K, Gradalski T, Szygula Z, Partsch H. Activity with and without Arm Sleeves: Compliance and Quality of Life After Breast Cancer Surgical- A Randomized Controlled Trial. LYMPHATIC RESEARCH AND BIOLOGY, 2018, Jan; 16 (3).

23. Ganz P. Breast Cancer, menopause, and longterm survivorship: Critical issues for the $21 \mathrm{st}$ century. Am J Med, 2005; 118 (12B): 1365-415.

24. Hormes JM, Lytle LA, Gross CR, Ahmed RL, Troxel AB, Schmitz KH. The Body Image and Relationships Scale: Development and Validation of a Measure of Body Image in Female Breast Cancer Survivors. J Clin Oncol, 2008, Mar; 26 (8): 1269-74.

25. Didem K, Ufuk YS, Serdar S, Zumre A. The comparison of two different physiotherapy methods in treatment of lymphedem after breast surgery. Breast Cancer Res Treat, 2005; 93: 49-
54.

26. Speck RM, Gross CR, Hormes JM, Ahmed RL, Lytle LA, Hwange WT, Schmitz KH. Changes in the body image and relationship scale following a one-year strength training trial for breast cancer survivors with or at risk for lymphedema. Breast cancer Res treat, 2010, Jun; 121 (2): 421-30.

27. Irdesel J, Celiktas SK. Effectiveness of exercise and compression garments in the treatment of breast cancer related lymphedema- Original article. Turk J Phys Med Rehab, 2007; 53: 16-21.

28. Akbari Haghighinejad H, Hadi N, Banihashemi $\mathrm{S}$, Abdinejad M, Tahmasebi S, et al. The Effect of Complex Decongestive Therapy on Post-Breast Surgery Quality of Life in Breast Cancer Patients With Unilateral Lymphedema and Its Predictive Factors, Shiraz E-Med J. 2016; 17 (1): e29200.

29. Pruzinsky T. Enhancing quality of life in medical populations: a vision for body image assessment and rehabilitation as standards of care. Body Image, 2004; 1: 71-81.

30. Passik SD, McDonald MV. Psychosocial aspects of upper extremity lymphedema in women treated for breast carcinoma. Cancer, 1998; 83: 2817-20.

31. Passik S, Newman M, Brennan M, Holland J. Psychiatric consultation for women undergoing rehabilitation for upper extremity lymphedema following breast cancer treatment. $\mathrm{J}$ Pain Symptom Manage, 1993, May; 8 (4): 226-33.

32. Barton D, Brooks T, Cieslak A, Elkins G, Clark $\mathrm{P}$, Baydoun M, et al. Preliminary evaluation of a mind-body intervention to improve body and/or self-image: A phase II randomized trial. Cancer Res, 2019, Feb; 79 (4). 